\title{
EDITORIAL
}

\section{¿Quién puede ser autor de un artículo científico? ¿Yo, tú, el, ellos, nosotros? ¿Quién sabe?}

\author{
¿Who can be author of a scientific paper? \\ ¿Me, you, him, them, us? ¿Who knows?
}

De acuerdo con el diccionario de la Real Academia Española (1), autor tiene cinco acepciones, pero la que se acerca a los intereses del presente escrito reza así: "Persona que ha producido alguna obra científica, literaria o artística". No obstante, en el ámbito científico y académico esta definición puede ser más amplia, compleja y aun alcanzar diferentes connotaciones si se tienen en cuenta algunas reflexiones generales sobre la materia y no menos importante cuando se tiene presente; como debería ser, las razones éticas para firmar como autor o coautor de un artículo. Estas consideraciones son las intrínsecas de la investigación y que se generan dentro de un grupo en particular, que se preocupa, por ejemplo, en ser el primero en publicar artículos sobre un tema específico. Otros, con otra óptica, podrían tener interés en ostentar el mayor número de artículos publicados en una determinada área del conocimiento.

Pero también resulta importante tener en cuenta factores colaterales que ejercen presión bien sea a través de instituciones, empresas, grupos de investigación, así como exigencias para mejoras laborales y consecución de recursos económicos ante entidades financiadoras de la investigación. De todas las presiones antes citadas; que no son todas, han resultado un sinnúmero de estrategias promovidas por los investigadores, tanto internas como externas al grupo al cual pertenecen. Por supuesto, también se incluyen las estrategias interinstitucionales tanto nacionales como internacionales, lo cual agrega un ingrediente más en la complejidad de definir autores, y en especial, su orden de aparición en la publicación de los artículos. En todos los casos, se debe precisar quien es autor y quien o quienes aparecerán en la sección de agradecimientos, que a nuestro modo de ver las cosas, esta sección siempre deberá estar presente en los manuscritos.

El solo hecho de definir quién será el primer autor (que en general se considera el de mayor importancia o quien hace la mayor parte del trabajo), y desde luego, el subsiguiente orden de aparición de coautores, puede resultar complejo en ciertos casos o extremadamente sencillo cuando se recurre a la subjetividad acompañada de visos dictatoriales del líder del grupo. El último autor, usualmente es el investigador senior quien supervisa el trabajo y es el autor corresponsal.

Por ejemplo, definir quiénes serán los firmantes y por lo tanto, responsables del contenido de un artículo puede resultar "relativamente sencillo" cuando se escribe un proyecto de investigación institucional con un mínimo de participantes y además, financiado con recursos propios. Pero, seguramente no resultará igual de sencillo definir autor y coautores cuando la investigación que se realiza es multidisciplinaria, interinstitucional y adicionalmente se cuenta con la participación de organismos internacionales, presentándose en estos casos los llamados "artículos multiautoriales" (2). 
Por referirnos a un solo caso de abril del 2011 y sin mayor esfuerzo de búsqueda, citamos el artículo "Fever with thrombocytopenia associated with a novel Bunyavirus in China", el cual aparece firmado por 46 autores (3). Si bien pueden surgir varios interrogantes, creemos que una pregunta importante seria: ¿Cómo hicieron ellos para definir el orden de la aparición en el manuscrito? Por supuesto no lo sabemos, pero en definitiva, lo respetamos, aceptamos y seguramente ellos tendrán múltiples razones que lo justifiquen. Debemos dejar bien claro que en ningún momento cuestionamos absolutamente nada del contenido de dicho manuscrito y mucho menos de la prestigiosa revista en donde fue publicado.

Por otra parte, resulta difícil concebir que un solo autor pueda adelantar en su totalidad un trabajo de investigación. Se requiere de un equipo de trabajo en donde estarán colegas, colaboradores, técnicos de laboratorio, trabajadores de campo, estadísticos, ingenieros de sistemas, traductores, secretarias, dibujantes, etc. Por supuesto, no todos serán autores, pero habrá que dejar definida su participación desde el inicio de la investigación. Este comentario cobra importancia cuando hoy se sugiere que se constituyan equipos interdisciplinarios, interinstitucionales y ojalá con vinculación de investigadores del extranjero, lo cual lógicamente aumentará el número de autores por artículo publicado.

También es cierto que dependiendo del tipo de artículos, tales como casos clínicos, revisiones de literatura y de temas, dadas sus características particulares y estructurales de desarrollo en el tiempo, presentan menos dificultades a la hora de decidir quiénes pueden firmar como autores de acuerdo con su porcentaje o grado de participación.

Estas complicaciones pueden resolverse en gran medida, si desde el inicio se tienen en cuenta algunos principios para definir la participación de todos los integrantes de la investigación, tal como lo propone Acosta (4), quien sugiere la aplicación de un formato que contempla la utilización de criterios cuantitativos en las diferentes fases de desarrollo del proyecto de investigación. La clave en este caso es que todos los investigadores deberán diligenciar detalladamente el formato. También se deberán considerar por otra parte, los aspectos éticos cuando se trate de investigaciones con seres humanos y animales, teniendo en cuenta la reglamentación mundial de estos temas, así como la interna de instituciones y leyes específicas de cada país en donde se genera la investigación (5).

Asimismo existen otras alternativas que necesariamente tienen que tenerse en cuenta en revistas biomédicas y que para el caso de nuestra revista aplica y son las Normas de Estilo Vancouver (5) en donde se puede consultar en detalle cada componente de un artículo científico, incluidas las condiciones de autoría de los mismos. Lo importante es que se tengan en cuenta las normas, requisitos y en definitiva se pongan en práctica en el momento cierto.

Con relación a la publicación de 294 artículos en la Revista MVZ Córdoba en el periodo 2000-2010, el número de autores promedio por artículo fue de 3.2, lo cual está lejos de ser considerado dentro de la clasificación de artículos multiautoriales (Figura 1). No negamos que la autoría múltiple es un fenómeno que está presente, pero debe estar limitado solo para aquellos casos estrictamente necesarios y plenamente justificados. Si bien no existe un consenso, es recomendable, insistimos, tener en cuenta las Normas de Vancouver (5) que innegablemente han contribuido con los mismos investigadores e instituciones a plantear reglamentaciones internas en sus grupos de investigación.

Como cualquier mortal, fácilmente se puede caer en la tentación de cuestionar en los corrillos la participación de autores en un artículo, pero antes, se debe aprender a 


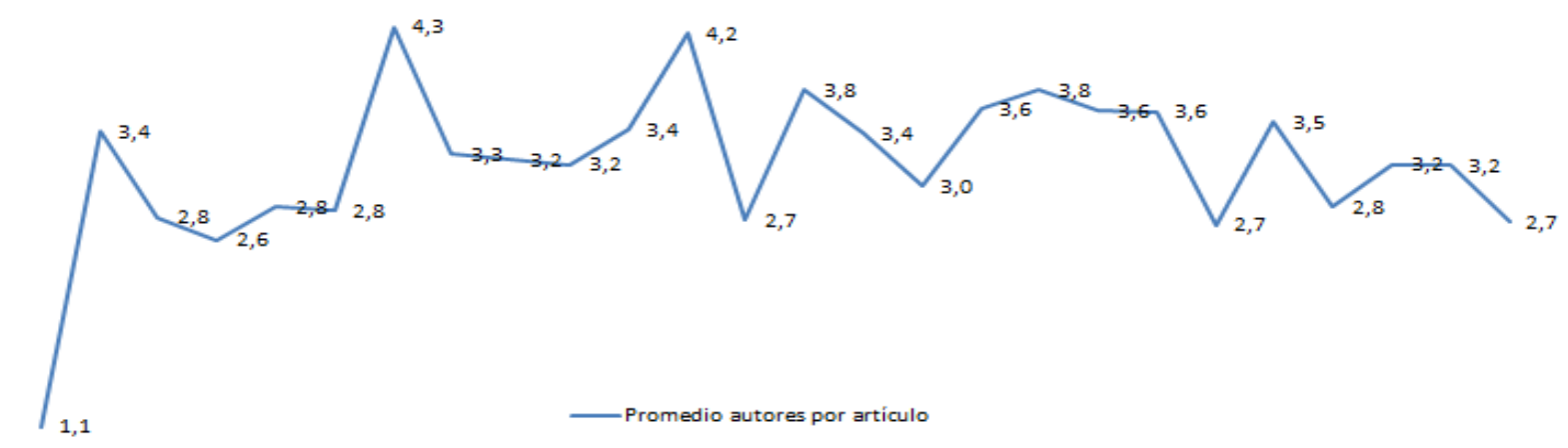

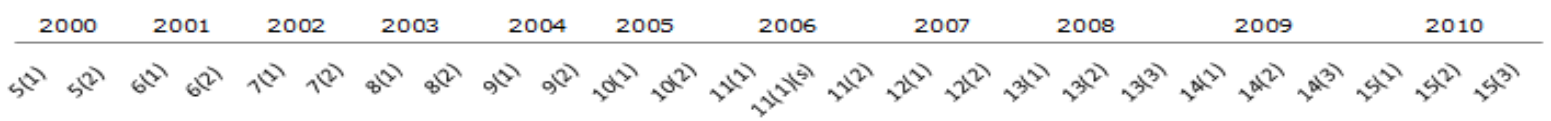

Figura 1. Promedio de autores por articulo en la Revista MVZ Córdoba periodo 2000-2010.

ser autores, justamente participando en la formulación, presentación de resultados, discusión y escritura del manuscrito, y luego vivir la experiencia del sometimiento de ese escrito a la evaluación crítica y anónima de árbitros, quienes tendrán de alguna manera la última palabra. También se puede optar por consultar la literatura pertinente e instruirse a fin de evitar conciliábulos y dictaminar con ligereza quien puede ser autor de un artículo, si él, ellos o nosotros. Finalmente, sólo aquel individuo que publica y consulta, sabrá con certeza quién puede ser autor. Escribamos entonces y no cuestionemos. Quedan formalmente invitados.

\section{REFERENCIAS}

1. Diccionario de la lengua española. Vigésima segunda edición. Autor, ra. Real Academia Española. [En Linea]. 2001. [Fecha de acceso, enero de 2011] URL Disponible en: http://buscon. rae.es/draeI/SrvltConsulta?TIPO_ $B U S=3 \& L E M A=A U T O R$

2. Silva GA. La autoría múltiple y la autoría injustificada en los artículos científicos. La autoría múltiple sobrecarga el sistema de diseminación de información científica y a veces sirve de parapeto a la concesión indebida del crédito del autor. Investigación en Salud 2005; VII(2). URL Disponible en: http://www.sld.cu/galerias/pdf/ uvs/cirured/autoria_multiple.pdf
3. Xue-Jie Yu, Mi-Fang Liang, Shou-Yin Zhang, Yan Liu, Jian-Dong Li, Yu-Lan Sun, et al. Fever with Thrombocytopenia Associated with a Novel Bunyavirus in China. N Engl J Med 2011; 364:1523-1532.

4. A. Acosta. Cómo definir autoría y orden de autoría en artículos científicos usando criterioscuantitativos. Universitas Scientiarum 2007; 12(1): 67-81.

5. González GC. Requisitos de Uniformidad para Manuscritos enviados a Revistas Biomédicas. [En línea]. La Coruña: Biblioteca del Complexo Hospitalario Universitario de A Coruña; 2006 [acceso febrero de 2011]. URL Disponible en: http://www. fisterra. com/recursos_web/mbe/vancouver. asp\#Autoría 